\title{
Nonlinear Desingularization in Certain Free-Boundary Problems $\star$
}

\author{
M. S. Berger ${ }^{1}$ and L. E. Fraenkel ${ }^{2}$ \\ 1 Department of Mathematics and Statistics, University of Massachusetts, Amherst, MA 01003, USA, \\ and \\ 2 Mathematics Division, University of Sussex, Brighton BN1 9QH, England
}

\begin{abstract}
We consider a nonlinear, elliptic, free-boundary problem involving an initially unknown set $A$ that represents, for example, the cross-section of a steady vortex ring or of a confined plasma in equilibrium. The solutions are characterized by a variational principle which allows us to describe their behaviour under a limiting process such that the diameter of $A$ tends to zero, while the solutions degenerate to the solution of a related linear problem. This limiting solution is the sum of the Green function of the linear operator and of a smooth function satisfying the boundary conditions. Mathematically speaking, this limiting process, that we call "nonlinear desingularization", is a novel kind of bifurcation phenomenon since the nonlinear effect here involves smoothing the singularity of the associated linear problem.
\end{abstract}

"Nonlinear desingularization" is an interesting, hitherto little studied phenomenon in nonlinear partial differential equations. By nonlinear desingularization we mean that a linear boundary-value problem, whose solution possesses one or more isolated singularities, is a degenerate form of a family of nonlinear problems whose solutions are smooth and, moreover, converge to the solution of the linear system upon degeneration. This phenomenon occurs, for example, in the study of vortex motion in ideal fluids; there a circular vortex filament is used to approximate a steady vortex ring of small cross-section. The Stokes stream function of the filament is the Green function of an elliptic operator, and hence has an isolated singularity, while the Stokes stream function for the vortex ring of small crosssection satisfies a non-linear partial differential equation, and is smooth. In this paper we study this situation from a global point of view that is independent of the elaborate singular-perturbation techniques often used for such problems.

Nonlinear desingularization arises in problems of theoretical physics other than the classical problem mentioned above. For example, it occurs in the onset of flux penetration, as determined by the Ginzberg-Landau theory, in supercon-

* Research partially supported by A FOSR and NSF grants 
ductors of type II (see [1]). Adler [2] has discussed it in connection with new classes of static, Euclidean, SU(2) Yang-Mills fields (monopoles) that are not spherically symmetric and have unit Pontrjagin index. We hope to consider these applications in a later paper.

\section{Introduction}

The basic ideas of this paper can be illustrated by means of a relatively simple example in one dimension. Consider the problem of finding simultaneously a function $U$ and an open set $A \subset(0,1)$ such that

$$
\begin{aligned}
-U_{x x} & =\left\{\begin{array}{lll}
\lambda & \text { in } & A, \\
0 & \text { in } \quad(0,1) \backslash \bar{A},
\end{array}\right. \\
\left.U\right|_{\partial A} & =0, \quad U_{x} \text { continuous on } \partial A, \\
U(0) & =-c, \quad U(1)=-1-c,
\end{aligned}
$$

where $\lambda$ and $c$ are given (strictly) positive parameters, and $U_{x}=d U / d x$. Note that (1.1a) and (1.1c) imply that $U(x)>0$ in $A$. We can interpret $y=U(x)$ as the equation of a (static) string subject to an upward loading $\lambda$ (force/length) that is applied only at points where $U(x)>0$, the ends of the string being fixed at the points $(0,-c)$ and $(1,-1-c)$.

As it happens, (1.1) can be solved explicitly, but in this it is exceptional, and we first ask : is there a linear problem that yields approximations to solutions $(U, A)$ of (1.1)? The answer is Yes, but only for a limiting situation as $\lambda \rightarrow \infty$.

Assume that the set $\bar{A}$ tends to a point, say $a$, as the "loading" $\lambda \rightarrow \infty$; then the relevant linear problem is

$$
\left.\begin{array}{rl}
-V_{x x} & =h \delta(x-a) \quad \text { in }(0,1), \\
V(0) & =-c, \quad V(1)=-1-c,
\end{array}\right\}
$$

where $\delta$ denotes the Dirac distribution; the constants $h$ and $a$ are still to be determined. The solution is

$$
V(x)= \begin{cases}-c+(h-h a-1) x, & 0 \leqq x \leqq a, \\ -1-c+(h a+1)(1-x), & a \leqq x \leqq 1 .\end{cases}
$$

The assumption $\bar{A} \rightarrow\{a\}$ means that, in the limit, $\max V(x)=V(a)=0$, which determines $h(a)$. In this particular case, the value $a$ can then be determined from the condition that it minimizes the elastic energy $\frac{1}{2} \int_{0}^{1} V_{x}^{2} d x$ of the string. Accordingly,

$$
h=\frac{c+a}{a(1-a)}, \quad a=\frac{c}{2 c+1} .
$$

Equation (1.3) can be written in a form that extends to other problems:

$$
V(x)=h G(x, a)-q(x), \quad q(x)=c+x,
$$

where $G$ is the Green function of the Dirichlet problem for the operator $-d^{2} / d x^{2}$, and $-q$ is the solution of $-q_{x x}=0$ that satisfies the boundary conditions. 
How can we find the precise relationship between the solutions of a nonlinear problem like (1.1) and the solution of a linear model like (1.2)? Two conceivable ways are (a) to seek a solution of the full problem by adding small perturbations to the solution of the linear problem, (b) to characterize and bound the solutions of the nonlinear problem globally, and then to analyze their behaviour as $\lambda \rightarrow \infty$. In this paper we adopt the second method, which is in accord with our ideas on bifurcation in [4], there called the "method of nonlinear descent". For the partial differential equations to be considered, we shall use an isoperimetric variational principle for functions in a Sobolev space. However, for the simple problem above, we can relate (1.5) to the explicit solutions of (1.1), which we now describe.

Since $U_{x x}<0$ in $A$, the set $A$ is a single subinterval of $(0,1)$, and we let $A=(a, b)$. First we solve separately in the intervals $[0, a),(a, b)$, and $(b, 1]$; then we impose continuity of $U$ and $U_{x}$ at $x=a, b$. It turns out that, for prescribed $\lambda$, there is no solution if $\lambda<\lambda_{0}=8(2 c+1)$, and one solution if $\lambda=\lambda_{0}$. For $\lambda>\lambda_{0}$ there are two solutions $U_{1}$ and $U_{2}$ as in Fig. 1, with

$$
\begin{aligned}
& a_{1}, a_{2}=\frac{c}{2(2 c+1)}\left\{1 \pm\left(1-\frac{\lambda_{0}}{\lambda}\right)^{1 / 2}\right\} \text { respectively, } \\
& b_{1}, b_{2}=\frac{1}{2(2 c+1)}\left\{(3 c+1) \mp(c+1)\left(1-\frac{\lambda_{0}}{\lambda}\right)^{1 / 2}\right\} \text { respectively. }
\end{aligned}
$$

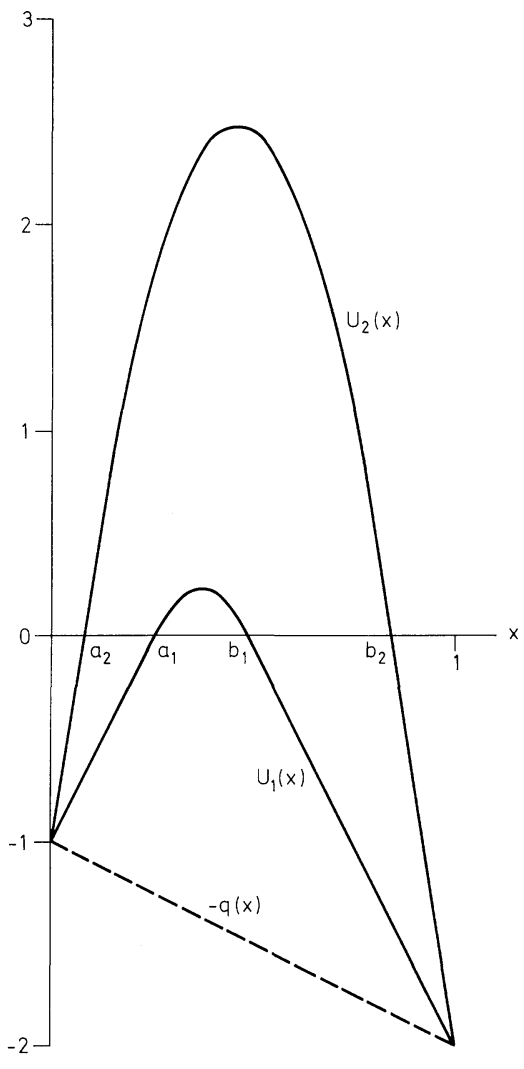

Fig. 1. The solutions $U_{1}$ and $U_{2}$ of (1.1) for prescribed $\lambda>\lambda_{0}$. The case drawn is $c=1$ (for which $\lambda_{0}=24$ ) and $\lambda / \lambda_{0}=1.4$ 
Writing

$$
U_{j}(x)=u_{j}(x)-q(x) \quad(j=1,2),
$$

where $q$ is as in (1.5), we find that

$$
u_{j}(x)=\left\{\begin{array}{l}
\frac{c+a_{j}}{a_{j}} x, \quad 0 \leqq x \leqq a_{j}, \\
\frac{c+a_{j}}{a_{j}} x-\frac{1}{2} \lambda\left(x-a_{j}\right)^{2}, \quad a_{j} \leqq x \leqq b_{j}, \\
\frac{c+b_{j}}{1-b_{j}}(1-x), \quad b_{j} \leqq x \leqq 1 .
\end{array}\right.
$$

From this it is clear that $a_{1}(\lambda) \rightarrow c /(2 c+1), b_{1}(\lambda) \rightarrow c /(2 c+1)$, and $U_{1}(x, \lambda) \rightarrow V(x)$ as $\lambda \rightarrow \infty$.

One important point remains. In the variational formulation we shall prescribe, in place of $\lambda$, a parameter $\mu$ that in the present case is defined by

$$
\mu=\int_{A} U(x) d x ;
$$

then $\lambda(\mu)$ is calculated a posteriori, and nonlinear desingularization occurs as $\mu \rightarrow 0$. Here, this has the additional advantage that (1.1) has exactly one solution for each prescribed $\mu \in(0, \infty)$; it is $\left(U_{1}, a_{1}, b_{1}\right)$ for $\mu \leqq \mu_{0}=\lambda_{0} / 96$, and $\left(U_{2}, a_{2}, b_{2}\right)$ for $\mu>\mu_{0}$. A calculation shows that $\lambda(\mu)$ is defined (inversely) by

$$
\mu=\frac{\lambda}{96}\left\{1-\left(1-\frac{\lambda_{0}}{\lambda}\right)^{1 / 2}\right\}^{3} \text { for } \mu \leqq \mu_{0},
$$

and

$$
\mu=\frac{\lambda}{96}\left\{1+\left(1-\frac{\lambda_{0}}{\lambda}\right)^{1 / 2}\right\}^{3} \text { for } \mu \geqq \mu_{0} ;
$$

the situation is depicted in Figs. 2 and 3.

The primary reason for introducing $\mu$, however, is that $\mu$ characterizes the set, in a Sobolev space, over which we shall minimize the Dirichlet form of our problem in order to obtain solutions. These are also solutions of an unconstrained variational problem (that is, critical points of a certain functional considered on the whole Sobolev space) but in that formulation they are saddle points rather than extrema, making quantitative estimates much more elusive.

We shall obtain results of the foregoing kind, necessarily by less explicit methods and therefore in less detail, for a general free-boundary problem involving a semi-linear elliptic equation of order two, in a bounded domain $\Omega \subset \mathbb{R}^{2}$. Section 2 contains various formulations of this problem, and describes some physical applications. In Sect. 3 we present a simple example in two space dimensions, and state our objective and method more precisely. In Sect. 4 we solve the variational form of the problem, and obtain the rather sharp estimates needed for the limiting procedure; this leads to a demonstration, in Sect. 5, of the nonlinear desingularization process for the problem at hand. 

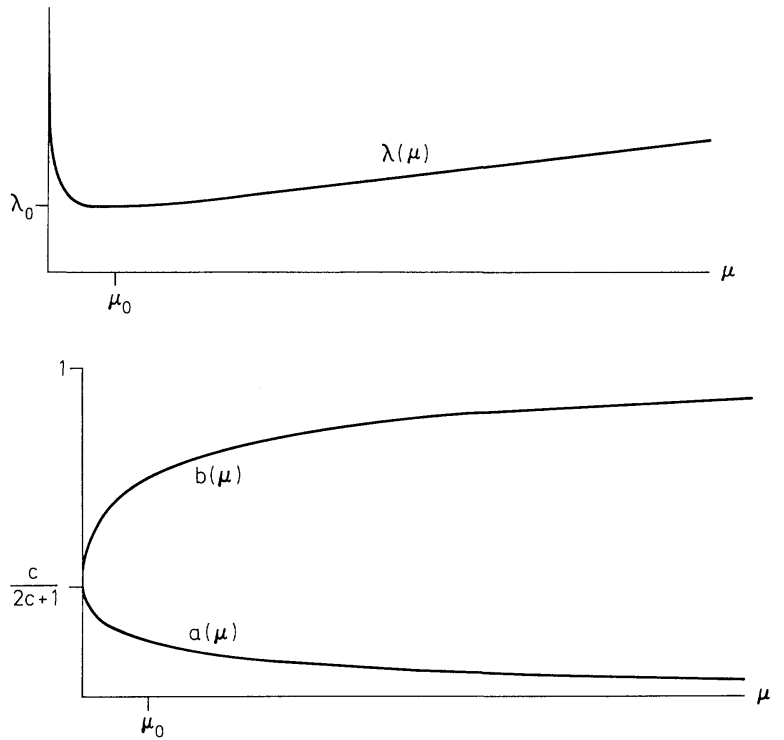

Fig. 2. Form of the function $\lambda(\mu)$ for the problem (1.1). The case drawn is $c=1\left(\lambda_{0}=24, \mu_{0}=\frac{1}{A}\right)$

Fig. 3. Form of the functions $a(\mu)$ and $b(\mu)$ defining the set $A$ in (1.1). The case drawn is $c=1$

\section{Formulations of the Problem}

\subsection{The Free-Boundary Problem}

Let $\Omega$ be a bounded domain (a bounded open connected set) in the plane $\mathbb{R}^{2}$, and let $L$ be a formally self-adjoint, uniformly elliptic operator of the second order:

$$
L=-\sum_{i, j=1}^{2} \frac{\partial}{\partial x_{i}}\left\{a_{i j}(x) \frac{\partial}{\partial x_{j}}\right\},
$$

where the matrix $\left(a_{i j}(x)\right)$ is symmetric and positive definite on $\bar{\Omega}$, and each $a_{i j} \in C^{\infty}(\bar{\Omega})$. We consider the free-boundary problem

$$
\begin{aligned}
& L \Psi= \begin{cases}\lambda f(x, \Psi) & \text { in } \quad A, \\
0 & \text { in } \quad \Omega \backslash \bar{A},\end{cases} \\
& \left.\Psi\right|_{\partial A}=0, \quad \operatorname{grad} \Psi \text { continuous on } \partial A, \\
& \left.\Psi\right|_{\partial \Omega}=-q_{0}(x, \kappa) \text {. }
\end{aligned}
$$

Here $f: \bar{\Omega} \times \mathbb{R} \rightarrow[0, \infty)$ and $q_{0}: \partial \Omega \times I \rightarrow(0, \infty)$ are given functions, specified more precisely in Sect. $2.4(I \subset \mathbb{R}$ is an interval housing the parameter $\kappa) ; \lambda$ is a real positive parameter which, for the moment, we regard as prescribed; and the boundary $\partial A$, of the open set $A$, is "free" in that it cannot be chosen a priori, but forms a part of the solution.

The set $A$ may be visualized as the cross-section of a steady vortex ring $[6,8]$ (see Appendix A for detailed comments), or of one member of a steady planar vortex pair $[9,12]$. The problem in which $A$ represents the cross-section of a confined plasma in equilibrium $[9,15]$ is only slightly different. In fact, if we set $u=-\Psi$ in the problems on pp. 52 and 59 of [15], and choose to prescribe the 
boundary-value constant $\gamma$ there, and to compute the total current a posteriori, then those problems assume the form (2.1). The total current in the plasma, denoted by $I$ in [15], corresponds to the circulation of a vortex ring, and to the function $h$ in (3.9) of the present paper; the change of formulation just mentioned for the plasma was adopted for vortex rings when passing from the perturbation theory for rings of small cross-section [6] to the global theory [8].

We allow $q_{0}$ to depend on the parameter $\kappa$ because more interesting cases arise as $\lambda \rightarrow \infty$ if $q_{0}$ is allowed to vary than if $q_{0}$ is fixed. In fact, we shall see (after Theorem 5.2) that, if $q_{0}$ is fixed, then the total-current or circulation function $h$ tends to zero.

\subsection{The Associated Semi-Linear Dirichlet Problem}

As in [8], the difficulty of the free boundary is removed by the following artifice. The maximum principle implies that $\Psi(x)>0$ in $A$ and that $\Psi(x)<0$ in $\Omega \backslash \bar{A}$. Therefore we (a) re-define $f$, if necessary, so that $f(x, t)=0$ for $t<0$, (b) confine attention to Hölder-continuous functions $f$. (In [8], the continuity of $f$ is ultimately relaxed, but in the present paper our result depends on the connectedness of the set $A$, which we cannot prove without strong restrictions on $f$.) Then the free-boundary problem (2.1) reduces to the semi-linear Dirichlet problem

$$
\begin{aligned}
L \Psi & =\lambda f(x, \Psi) \quad \text { in } \Omega, \\
\left.\Psi\right|_{\partial \Omega} & =-q_{0}(x, \kappa) .
\end{aligned}
$$

We now transform this problem into one with a homogeneous boundary condition. The problem

$$
L q=0 \quad \text { in } \Omega,\left.\quad q\right|_{\partial \Omega}=q_{0}(x, \kappa),
$$

has a unique solution $q(x, \kappa)$, and we define

$$
\psi=\Psi+q \text {. }
$$

Then (2.2) becomes

$$
\begin{aligned}
L \psi & =\lambda f(x, \psi-q) \text { in } \Omega, \\
\left.\psi\right|_{\partial \Omega} & =0
\end{aligned}
$$

The Sobolev space $\stackrel{\circ}{W}_{p}^{1}(\Omega), 1 \leqq p<\infty$, may be defined as the completion of the set $C_{0}^{\infty}(\Omega)$ (of infinitely differentiable, real-valued functions having compact support in $\Omega$ ) in the norm

$$
\|u\|_{1, p}=\left\{\int_{\Omega}|\nabla u|^{p}\right\}^{1 / p}
$$

here the integration element $d x=d x_{1} d x_{2}$ is implied, and an elementary calculation shows that, if $\Omega$ is contained between parallel lines distance $l$ apart, then

$$
\left\{\int_{\Omega}|u|^{p}\right\}^{1 / p} \leqq \frac{1}{2} p^{-1 / p} l\|u\|_{1, p}
$$


For the case $p=2$ of a Hilbert space, we shall abbreviate $\stackrel{\circ}{W}_{2}^{1}(\Omega)$ to $W$, and $\|\cdot\|_{1,2}$ to $\|\cdot\|$; this norm may be called the Dirichlet norm. We introduce the bilinear form corresponding to the operator $L$ :

$$
B_{L}(u, v)=\sum_{i, j=1}^{2} \int_{\Omega} a_{i j}(x) \frac{\partial u}{\partial x_{i}} \frac{\partial v}{\partial x_{j}}, \quad B(u)=B_{L}(u, u),
$$

for all $u$ and $v$ in $W$. Then $\psi$ is a generalized solution of $(2.4 \mathrm{a}, \mathrm{b})$ if $\psi \in W$ and

$$
B_{L}(\phi, \psi)=\lambda \int_{\Omega} \phi f(x, \psi-q) \text { for all } \phi \in W
$$

\subsection{A Variational Principle}

In order to characterize the desired family of solutions, we now formulate an isoperimetric variational principle as follows. Define the functional $J: W \times I \rightarrow[0, \infty)$ by

$$
J(u, \kappa)=\int_{\Omega} F(x, u-q), \quad \text { where } F(x, t)=\int_{0}^{t} f(x, s) d s,
$$

and where $u-q=u(x)-q(x, \kappa)$. Here the integral over $\Omega$ need be taken only over the set

$$
A_{u}=\{x \in \Omega \mid u(x)>q(x, \kappa)\},
$$

which becomes the desired set $A=A_{\psi}$ in (2.1) when $u=\psi$; a similar remark applies to (2.5). As we show below, (2.5) characterizes a critical point (or stationary point) $\psi$ of the restriction of the quadratic functional $B$ to the set

$$
\sigma(\mu, \kappa)=\{u \in W \mid J(u, \kappa)=\mathrm{const}=\mu>0\},
$$

which may be regarded as a surface in $W$. However, we are not free to prescribe both $\lambda$ and $\mu$; we choose to prescribe $\mu$ and to compute $\lambda=\lambda(\mu, \kappa)$ a posteriori. If

$$
\psi \in \sigma(\mu, \kappa)\left(\text { that is, } \psi \in W \text { and } J(\psi, \kappa)=\int_{\Omega} F(x, \psi-q)=\mu>0\right)
$$

and (2.5) holds, then the pair $(\psi, \lambda)$ will be called a generalized solution of (2.4). Obviously (2.4) means $(2.4 \mathrm{a}-\mathrm{c})$; we solve this problem by means of the variational principle

$$
\min _{u \in \sigma(\mu, \kappa)} B(u)
$$

then (2.5), with $\phi=\psi$, implies that

$$
0<\lambda(\mu, \kappa)=B(\psi) / \int_{\Omega} \psi f(x, \psi-q)
$$

The variational principle (2.6) is not precisely the analogue of that used in [8], but in Appendix B we prove a certain equivalence of the reciprocal variational principles

$$
\max _{B(u)=\eta} J(u, \kappa) \text { and } \min _{J(u, \kappa)=\mu} B(u),
$$

the former being the analogue of that in [8]. 


\subsection{Precise Conditions on the Data}

(i) The assumptions in Sect. 2.1 about the operator $L$ imply the existence of constants $\beta_{1}$ and $\beta_{2}$ such that $0<\beta_{1} \leqq \beta_{2}$ and

$$
\beta_{1}\|u\|^{2} \leqq B(u) \leqq \beta_{2}\|u\|^{2} \text { for all } u \in W .
$$

Here $\|\cdot\|$ is the Dirichlet norm introduced after $(2.4 \mathrm{~b})$.

(ii) We assume that $f \in C^{1}(\bar{\Omega} \times \mathbb{R})$, that $f(x, t)=0$ for $t \leqq 0$, that $f(x, t)>0$ for $t>0$, and that $f(x, \cdot)$ is convex for each fixed $x \in \bar{\Omega}$. This implies, of course, that $f(x, t)$ is a strictly increasing function of $t$ for $t \geqq 0$. Moreover, we suppose that

$$
M_{1} t^{m_{1}} \leqq f(x, t) \leqq K_{2} t+M_{2} t^{m_{2}}, \quad 1<m_{1} \leqq m_{2}, \quad t \geqq 0,
$$

where $m_{j}-1, M_{j}(j=1,2)$ and $K_{2}$ are positive constants (independent of $x$ and $t$ ). The requirements on the constant $m_{1}$ are needed for the validity of our estimates but can be relaxed in special cases. (See Remark below.)

(iii) The boundary $\partial \Omega$ is taken to be of class $C^{\infty}$. Regarding the boundary values $-q_{0}(x, \kappa)$ of $\Psi$, we assume that $q_{0}(\cdot, \kappa) \in C^{\infty}(\partial \Omega)$ for each fixed $\kappa$ in the interval $I \subset \mathbb{R}$, and that

$$
\text { where } \left.\begin{array}{c}
q_{1}(\kappa) \leqq q_{0}(x, \kappa) \leqq q_{2}(\kappa) \\
0<\gamma_{1} \leqq q_{1}(\kappa) \text { and } q_{2}(\kappa) / q_{1}(\kappa) \leqq \gamma_{2},
\end{array}\right\}
$$

$\gamma_{1}$ and $\gamma_{2}$ being independent of $\kappa$. Thus $q_{0}(x, \kappa)$ is bounded away from zero but not from infinity, although its growth must be of the same order for all $x \in \partial \Omega$. Applying the maximum principle to the Eq. (2.3) determining q, we observe that

$$
\text { in (2.10a), } q_{0} \text { may be replaced by } q \text {. }
$$

Remark. The particular, but important, function

$$
f(x, t)=\left\{\begin{array}{l}
0, \quad t \leqq 0, \\
g(x) t, t>0, \quad g \in C^{1}(\bar{\Omega}), \quad g(x)>0 \text { on } \bar{\Omega},
\end{array}\right.
$$

is also admissible for the validity of our results, even though this $f \notin C^{1}(\bar{\Omega} \times \mathbb{R})$. This function is important because it is a prototype in the context of planar vortex pairs $[9,12]$ and of a confined plasma $[9,15]$. It is admissible because (a) it allows a proof that the set $A$ is connected, (b) the estimates in Appendix $C$ and Theorem 4.4 remain valid. Just as we adapt Theorem $3 \mathrm{G}$ of [8] to prove, in Theorem 4.3 below, connectedness of $A$ for functions $f$ as in (ii), so we can adapt (and correct several inaccuracies in) the proof of Theorem 3.5 in [12] to prove connectedness of $A$ for the function $f$ in (2.11). Having noted this fact, we shall use the hypotheses in (ii) henceforth.

\section{Expected Behaviour of the Solution as $\mu \rightarrow 0$}

The problem $(2.4 \mathrm{a}, \mathrm{b})$ admits the trivial solution $\psi=0$, but non-trivial solutions must exceed the values $q(x, \kappa) \geqq \gamma_{1}>0$ somewhere in $\Omega$ (on the set that we call $A$ ); hence they cannot be small in the space $C(\bar{\Omega})$ and do not bifurcate from the trivial solution in the traditional manner. Rather, the example (1.1) leads us to expect 
that solutions of the variational problem (2.6), which satisfy $(2.4 \mathrm{a}-\mathrm{c})$, are related to the solution of a linear problem involving the operator $L$ by way of the Green function $G(x, y)$, defined for fixed $y \in \Omega$ as the solution of

$$
L G=\delta(x-y) \text { in } \Omega,\left.\quad G\right|_{x \in \partial \Omega}=0 .
$$

Here $\delta$ denotes the Dirac distribution; the formal self-adjointness of $L$ implies that $G(x, y)=G(y, x)$, and the maximum principle implies that $G(x, y)>0$ on $\Omega \times \Omega$.

We now consider a second simple example, this time in two dimensions but with spherical symmetry. This prepares the way for estimates that we make in Sect. 4, and at the same time shows explicitly how the qualitative features observed for (1.1) are repeated in two dimensions even though the Green function is now quite different. (For spherically symmetric solutions, the same is true in higher dimensions.)

Let $\mathscr{B}$ denote the unit ball $\left\{x \in \mathbb{R}^{2}|| x \mid=r<1\right\}$; we seek a spherically symmetric solution $(\Psi, A, \lambda)$ of the following, in which $\Delta$ denotes the Laplace operator.

$$
-\Delta \Psi=\left\{\begin{array}{l}
\lambda \text { in } A=\{x \mid \Psi(x)>0\}, \\
0 \text { in } \mathscr{B} \backslash \bar{A},
\end{array}\right.
$$

$\operatorname{grad} \Psi$ continuous on $\partial A,\left.\Psi\right|_{\partial \mathscr{B}}=-q(\kappa)<0$,

$$
\int_{A} \Psi=\mu>0 \text {. }
$$

Here $f$ is a step function and violates the hypotheses in Sect. 2.4, but it can be handled for the case of spherical symmetry. This symmetry and the maximum principle imply that $A$ must be a ball about the origin; let its radius be $\varrho$. Calculating as in Appendix $\mathrm{C}$, we find that

$$
\begin{aligned}
\Psi(x) & =\left\{\begin{array}{c}
\frac{1}{4} \lambda\left(\varrho^{2}-r^{2}\right), \quad 0 \leqq r \leqq \varrho, \\
-q \log \frac{r}{\varrho} / \log \frac{1}{\varrho}, \varrho \leqq r \leqq 1,
\end{array}\right. \\
\lambda & =2 q / \varrho^{2} \log \frac{1}{\varrho}, \\
B(\psi) \equiv\|\psi\|^{2} & =\frac{2 \pi q^{2}}{\log (1 / \varrho)}\left\{1+\frac{1}{4 \log (1 / \varrho)}\right\}, \\
\mu & =\frac{1}{4} \pi q \varrho^{2} / \log \frac{1}{\varrho}, \\
h & \equiv \lambda \pi \varrho^{2}=2 \pi q / \log \frac{1}{\varrho},
\end{aligned}
$$

where $q=q(\kappa)$ and $\psi=\Psi+q$. As before, we see from (3.4) that there exists a critical value $\lambda_{0}$ such that, for given $\lambda$, we have no solution, one solution or two solutions according as $\lambda<\lambda_{0}, \lambda=\lambda_{0}$ or $\lambda>\lambda_{0}$; while (3.6) shows that, for given $\mu \in(0, \infty)$, we have exactly one solution [see Lemma C.2, which gives bounds for $\varrho(\mu, \kappa)$ ]. We also observe, from (3.3) and (3.7), that

$$
\Psi(x)=h G(x, 0)-q \text { for } \varrho \leqq r \leqq 1,
$$


and, from (3.6) and (3.4), that $\varrho(\mu, \kappa) \rightarrow 0$ and $\lambda(\mu, \kappa) \rightarrow \infty$ as $\mu \rightarrow 0$ [even if we allow $\kappa$ to vary with $\mu$, since $\left.q(\kappa) \geqq \gamma_{1}>0\right]$.

Returning to the variational problem (2.6) for the general case, we now make the guess that, as $\mu=\int_{A} F(x, \psi-q) \rightarrow 0$, the set $\bar{A}$ tends to a point, say $a$, and that $\lambda \rightarrow \infty$. Then, formally, (2.4a) becomes

$$
L \psi=\lambda f(x, \psi-q) \sim h(\mu, \kappa) \delta(x-a),
$$

where

$$
h(\mu, \kappa)=\lambda \int_{A} f(x, \psi-q) .
$$

Accordingly, we expect that the solution $\psi$ of (2.6) has the behaviour

$$
\psi(x ; \mu, \kappa) \sim h(\mu, \kappa) G(x, a) \text { as } \mu \rightarrow 0, \quad x \neq a,
$$

although $\psi$ will be bounded on $A$ for each fixed $\mu$, in contrast to $G(\cdot, a)$. [In the particular cases of Appendix C, we can even so choose $\kappa=\kappa(\mu)$ and $q$ that $h(\mu, \kappa)$ is a positive constant, and $\psi(x)=$ const $G(x, 0)$ outside the shrinking set $A$.]

In Sects. 4 and 5 we shall confirm these conjectures, under the hypotheses in Sect. 2. Once existence of solutions, and connectedness of the set $A$, have been proved, the principal step results from the bounds

$$
2 \pi / \sinh ^{-1}\left(\frac{\operatorname{diam} \Omega}{\operatorname{diam} A}\right)<\operatorname{cap}(\bar{A}, \Omega) \leqq\|\psi(\cdot, \mu, \kappa)\|^{2} / q_{1}(\kappa)^{2}
$$

for the electrostatic capacity of $\bar{A}$ relative to $\Omega$. [This is defined immediately before (4.13).] The lower bound is derived in [7], for any connected compact set $K \subset \Omega$ having positive diameter, by means of Steiner symmetrization, successively about two particular perpendicular lines, and recognition of the minimizing configuration for given diameter ratio. The upper bound in (3.10), which follows from the definition of capacity and the fact that $\psi(x, \mu, \kappa) \geqq q_{1}(\kappa)$ on $\bar{A}$, is useful because comparison with a spherically symmetric solution will give us a fairly sharp upper bound for $\|\psi(\cdot, \mu, \kappa)\|$.

Higher-dimensional cases, $\Omega \subset \mathbb{R}^{N}$ with $N \geqq 3$, elude our estimates, essentially because the electrostatic capacity of a line segment is positive in two dimensions, which allows us to bound the diameter of $A$, but is zero in three or more dimensions. Fortunately, the free-boundary problems that we have in mind are set in $\mathbb{R}^{2}$.

\section{Existence, and Some Properties, of the Variational Solution}

In this section we show that the variational problem (2.6) has a solution $\psi$, which we proceed to study in some detail. The set

$$
A=\{x \in \Omega \mid \psi(x)>q(x, \kappa)\}
$$

is of particular interest. 
Theorem 4.1. The variational problem $\min _{u \in \sigma(\mu, \kappa)} B(u)$ has a solution $\psi$ such that

(a) the pair $(\psi, \lambda)$ is a generalized solution of (2.4) when $\lambda$ is defined by (2.7),

(b) $\psi \in C^{2+\alpha}(\bar{\Omega})$ for any Hölder exponent $\alpha \in(0,1)$,

(c) $\psi(x)>0$ in $\Omega$.

Proof. Since the variational competition is at a fixed value of the parameter $\kappa$ in $q$, we omit display of $\kappa$ in this proof.

(i) First we show that, for any set of data as in Sect. 2, the set $\sigma(\mu)$ is not empty. Take any function $v \in C_{0}^{\infty}(\Omega)$ with $v(x)>0$ somewhere in $\Omega$; if $t_{0}$ denotes the smallest value $t$ such that $t v(x)=q(x)$ somewhere in $\Omega$, then $J(t v)$ increases continuously from 0 to $\infty$ with $t \geqq t_{0}$ [because $F(x, s)$ so increases with $s \geqq 0$ ]; consequently there exists a unique value $t_{1}$ such that $J\left(t_{1}, v\right)=\mu>0$, and then $t_{1} v \in \sigma(\mu)$.

(ii) Let $b=\inf _{u \in \sigma(\mu)} B(u)>0$, and let $\left\{u_{n}\right\}$ be a sequence in $\sigma(\mu)$ such that $B\left(u_{n}\right) \rightarrow b$ as $n \rightarrow \infty$. This sequence is bounded in the Hilbert space $W$ [given any $v \in \sigma(\mu)$, we may suppose that $B\left(u_{n}\right) \leqq B(v)+1$, and then use (2.8)]; hence there exist a subsequence, say $\left\{\psi_{n}\right\}$, and an element $\psi \in W$ such that $\psi_{n} \rightarrow \psi$ weakly in $W$. Sobolev embedding, and the upper bounding function for $f$ in (2.9), ensure that $J$ is continuous with respect to weak convergence in $W$; therefore $J(\psi)$ $=\lim J\left(\psi_{n}\right)=\mu$, and so $\psi \in \sigma(\mu)$ and $B(\psi) \geqq b$. But, since $B_{L}$ satisfies the axioms of an inner product, a familiar theorem on weak convergence states that $B(\psi)$ $\leqq \lim \inf B\left(\psi_{n}\right)=b$. Accordingly, $B(\psi)=b$.

(iii) Since the functionals $B$ and $J$ are $C^{1}$ (in fact, they are smoother than this), a standard result of the calculus of variations ([5], p. 123) ensures the existence of numbers $\lambda_{1}$ and $\lambda_{2}$, not both zero, such that

$$
\lambda_{1} B_{L}(\phi, \psi)=\lambda_{2} \int_{\Omega} \phi f(x, \psi-q) \text { for all } \phi \in W .
$$

The choice $\phi=\psi$ shows that $\lambda_{1} / \lambda_{2} \neq 0$ and $\lambda_{2} / \lambda_{1} \neq 0$, and (2.5) follows, so that (a) is proved.

(iv) Regularity theory for generalized solutions of elliptic equations [3], and the fact that $f \in C^{1}(\widetilde{\Omega} \times \mathbb{R})$, imply that the equivalence class $\psi$ has a representative $\psi \in C^{2+\alpha}(\bar{\Omega})$ for all $\alpha \in(0,1)$. Since $\lambda f(x, \psi-q) \geqq 0$, the maximum principle ensures that $\psi(x)>0$ in $\Omega$. (That $\psi(x) \geqq 0$ almost everywhere in $\Omega$ is actually a consequence of the variational characterization, and can be proved without regularity theory and the maximum principle; cf. [8], p. 26.)

Lemma 4.2. Let $E$ be a component of the set $A$ in (4.1), and define

$$
B_{L, E}(u, v)=\sum_{i, j=1}^{2} \int_{E} a_{i j}(x) \frac{\partial u}{\partial x_{i}} \frac{\partial v}{\partial x_{j}}, \quad B_{E}(u)=B_{L, E}(u, u) .
$$

Then, with $\Psi=\psi-q$ and $\psi$ as in Theorem 4.1, we have

$$
B_{L, E}(\Psi, \psi)=B_{E}(\Psi)
$$

and

$$
B_{E}(\psi)=B_{E}(\Psi)+B_{E}(q)
$$


Proof. Since $\left.\Psi\right|_{\partial E}=0$ and $L q=0$,

$$
B_{L, E}(\Psi, q)=\int_{E} \Psi L q=0,
$$

which proves (4.2) because $B_{L, E}(\Psi, \psi)-B_{E}(\Psi)=B_{L, E}(\Psi, q)$, and proves (4.3) because

$$
B_{E}(\psi)=B_{E}(\Psi+q)=B_{E}(\Psi)+2 B_{L, E}(\Psi, q)+B_{E}(q) .
$$

Theorem 4.3. The set $A$ is connected.

Proof. Suppose the contrary, that $A$ has at least two components $E_{1}$ and $E_{2}$. With the notation $f^{\prime}(x, t)=\partial f(x, t) / \partial t$ and $\Psi=\psi-q$, we obtain a contradiction by considering the second variation $([5]$, p. 125)

$$
\delta^{2} B(\psi, v)=B(v)-\lambda \int_{\Omega} v^{2} f^{\prime}(x, \Psi)
$$

of $B$, in the direction $v$ along the surface $\sigma(\mu, \kappa)$, for any $v \in W \cap C(\bar{\Omega})$ such that

$$
B_{L}(v, \psi)=0 \text { and }\|v\|>0 .
$$

[The condition $v \in C(\bar{\Omega})$ makes it easy to show that the remainder of the Taylor series for $J(\psi+t v+\ldots, \kappa)$ is $o\left(t^{2}\right)$.] The proof now proceeds as in [8], p. 31; but we include the remaining steps because the present functionals look different, and for the sake of completeness.

Define $\Phi_{j}(j=1,2)$ by $\Phi_{j}(x)=\Psi(x)$ in $E_{j}$, and $\Phi_{j}(x)=0$ elsewhere. Then $\Phi_{j} \in W$ ([10], p. 50), and $\Phi_{j} \in C(\bar{\Omega})$ by the regularity of $\Psi$ and the definition of $E_{j}$. Set $v=c_{1} \Phi_{1}-c_{2} \Phi_{2}$; there exist positive constants $c_{1}$ and $c_{2}$ such that $B_{L}(v, \psi)=0$ because, by (4.2),

$$
B_{L}(v, \psi)=c_{1} B_{L}\left(\Phi_{1}, \psi\right)-c_{2} B_{L}\left(\Phi_{2}, \psi\right)=c_{1} B\left(\Phi_{1}\right)-c_{2} B\left(\Phi_{2}\right) .
$$

Also, $\Phi_{1}$ and $\Phi_{2}$ are orthogonal in $W$ (since $E_{1}$ and $E_{2}$ are disjoint), so that $\|v\|>0$. By (4.2) and the definition (2.5) of generalized solution,

$$
B\left(\Phi_{j}\right)=B_{L}\left(\Phi_{j}, \psi\right)=\lambda \int_{\Omega} \Phi_{j} f(x, \Psi)=\lambda \int_{E_{j}} \Psi f(x, \Psi)
$$

whence

$$
\begin{aligned}
\delta^{2} B(\psi, v) & =\sum_{j=1}^{2} c_{j}^{2}\left\{B\left(\Phi_{j}\right)-\lambda \int_{E_{J}} \Phi_{j}^{2} f^{\prime}(x, \Psi)\right\} \\
& =\lambda \sum_{j=1}^{2} c_{j}^{2} \int_{E_{J}}\left\{\Psi f(x, \Psi)-\Psi^{2} f^{\prime}(x, \Psi)\right\}
\end{aligned}
$$

The convexity of $f$ implies that $f(x, t)<t f^{\prime}(x, t)$ for $t>0$; hence $\delta^{2} B(\psi, v)<0$, which contradicts the minimizing property of $\psi$.

Remarks. 1. Henceforth we set $\kappa=\mu$, thus identifying the two parameters of the variational problem (2.6). To have done so earlier would have given a misleading impression of the level set $\sigma$, now characterized by $J(u, \mu)=\mu$ and denoted by $\sigma(\mu)$; to keep two distinct parameters in what follows, would lead to unhelpful complexity. Of course, we can still choose $q(\cdot, \mu)$ to be independent of $\mu$. 
2. The estimates which follow are prompted by the spherically symmetric cases in Appendix C, and involve the following notation. Let $b$ be the radius of a ball (or disk) $\mathscr{B}$ contained in $\Omega$ (the larger $b$, the better the estimates). We shall make a comparison with a spherically symmetric solution in the ball $\mathscr{B}$, and $b \varrho(\mu)$ will be an estimate for the radius of the set $A$ of that comparison solution. The functions $\varrho$ and $g$ which follow may be regarded as generalizations of the functions $\varrho$ and $2 \pi q^{2} / B(\psi)$ defined by (3.6) and (3.5) respectively [indeed, the present $\varrho$ and $g$ reduce to those functions if, ignoring the restriction $k>1$ in (4.4b), we set $k=\frac{1}{2}, b=1$ and $M_{1}=1$ there $]$.

With the notation of Sect. 2.4 for bounding constants and functions, define

$$
\varepsilon(\mu)=C \mu^{1 / 2} q_{2}(\mu)^{-k} \quad\left(q_{2}(\mu) \geqq \gamma_{1}>0\right),
$$

where

$$
k=\frac{1}{2}\left(m_{1}+1\right)>1, \quad C=\frac{1}{b}\left(\frac{2}{k}\right)^{k / 2}\left\{\frac{2 k(2 k+1)}{M_{1} \pi}\right\}^{1 / 2} .
$$

Let $\varrho(\mu)$ be the unique solution in $(0,1)$ of the equation (cf. Lemma C.2)

$$
\varrho\left(\log \frac{1}{\varrho}\right)^{-k}=\varepsilon(\mu)
$$

and let

$$
\begin{aligned}
& \varrho(\mu)=\varepsilon(\mu)\left\{\log \frac{1}{\varepsilon(\mu)}-k \log \log \frac{1}{\varepsilon(\mu)}\right\}^{k}, \\
& \varrho(\mu)=\varepsilon(\mu)\left\{\log \frac{1}{\varepsilon(\mu)}\right\}^{k} ;
\end{aligned}
$$

when $\mu$ is so small that $\varepsilon(\mu)<1 / e$ and $\bar{\varrho}(\mu)<1$, we have $\varrho(\mu)<\varrho<\bar{\varrho}(\mu)$. Finally, define

$$
g(\mu)=\log \frac{1}{\varrho(\mu)} /\left\{1+\frac{k}{2|\log \varrho(\mu)|}\right\} \equiv g_{*}(\varrho(\mu))
$$

and

$$
\underline{g}(\mu)=g_{*}(\bar{\varrho}(\mu))
$$

we note that $g_{*}$ is a decreasing function, so that $\varrho(\mu)<\bar{\varrho}(\mu)$ implies $g(\mu)>\underline{g}(\mu)$.

3. An asymptotic approximation to $g(\mu)$, for $\mu \rightarrow 0$, will help to clarify what follows. By (4.6a) and $(4.5 \mathrm{~b}, \mathrm{c})$

$$
g(\mu) \sim \log \frac{1}{\varrho(\mu)} \sim \log \frac{1}{\varepsilon(\mu)} \quad \text { as } \quad \mu \rightarrow 0 .
$$

Moreover, if $q_{2}(\mu) \sim$ const $\mu^{-a}$ or $q_{2}(\mu) \sim$ const $\{\log (1 / \mu)\}^{a}$ for some constant $a \geqq 0$ (a supposition that we make only for the moment, to clarify the nature of $g(\mu)$ ) then, by $(4.4 \mathrm{a})$,

$$
g(\mu) \sim \text { const } \log (1 / \mu) \quad \text { as } \quad \mu \rightarrow 0 .
$$


Theorem 4.4. With the foregoing notation, the solution $(\psi, \lambda)$ of $(2.6)$ and (2.7) has the following bounds; when $\mu$ is so small that $\varepsilon(\mu)<1 /$ e and $\varrho(\mu)<1$, the implicitly defined function $g$ may be replaced by the explicitly defined $\underline{g}$, given in(4.6b)above.Moreover

$$
\begin{aligned}
B(\psi) & \leqq 2 \pi \beta_{2} q_{2}(\mu)^{2} / g(\mu), \\
\delta & \equiv \frac{\operatorname{diam} A}{\operatorname{diam} \Omega}<\frac{1}{\sinh \{c g(\mu)\}}, \quad c=\frac{\beta_{1}}{\beta_{2} \gamma_{2}^{2}}, \\
\lambda & <2 \pi \beta_{2} q_{2}(\mu)^{2} / \mu g(\mu), \\
\frac{1}{\lambda} & <\operatorname{const} \delta^{2}\left\{1+\operatorname{const}\left(\sinh ^{-1} \frac{1}{\delta}\right)^{1 / 2}\right\}\left\{1+\operatorname{const} B(\psi)^{l}\right\}, l=\frac{1}{2}\left(m_{2}-1\right)>0 .
\end{aligned}
$$

Remark. Note the following implication of (4.6a) for the bounding function in (4.10):

$$
\frac{1}{\sinh \{c g(\mu)\}} \sim k_{0} \varrho(\mu)^{c} \quad \text { as } \quad \mu \rightarrow 0, \quad k_{0}=2 e^{k c / 2},
$$

where (4.5) shows how $\varrho(\mu) \rightarrow 0$. Also, in $(4.12), \delta^{2}\left(\sinh ^{-1} 1 / \delta\right)^{1 / 2}$ is an increasing function for all $\delta>0$, so that (4.10) may be used there.

Proof of Theorem 4.4. (i) Fixing $\mu$, we denote the variational problem (2.6) by $P(L, f, q, \Omega)$, and the set of admissible functions [previously called $\sigma(\mu, \kappa)$ ] by $S(f, q, \Omega)$; this allows us to indicate particular choices of $L, f, q$, and $\Omega$. We shall compare solutions $\psi$ and $\psi_{4}$ of the problems $P(L, f, q, \Omega)$ and $P\left(-\Delta, f_{1}, q_{2}, \mathscr{B}\right)$, respectively, for the same value of $\mu$; here $\Delta$ denotes the Laplace operator, $f_{1}$ the lower bounding function in (2.9), $q_{2}$ the upper bounding function in (2.10), and $\mathscr{B}$ a ball in $\Omega$.

Let $\psi_{2}$ be a solution of the problem $P\left(L, f, q_{2}, \Omega\right)$. Since $q \leqq q_{2}$, we have

$$
\int_{\Omega} F\left(x, \psi_{2}-q\right) \geqq \int_{\Omega} F\left(x, \psi_{2}-q_{2}\right)=\mu ;
$$

hence $t \psi_{2} \in S(f, q, \Omega)$ for some $t \in(0,1]$, and so $B(\psi) \leqq B\left(t \psi_{2}\right) \leqq B\left(\psi_{2}\right)$.

Let $\psi_{3}$ be a solution of the problem $P\left(-\Delta, f, q_{2}, \mathscr{B}\right)$. Extend it to $\Omega$ by defining $\psi_{3}(x)=0$ outside $\mathscr{B}$. Then $\psi_{3} \in S\left(f, q_{2}, \Omega\right)$, and so $B\left(\psi_{2}\right) \leqq B\left(\psi_{3}\right) \leqq \beta_{2}\left\|\psi_{3}\right\|^{2}$.

Let $\psi_{4}$ be a solution of the problem $P\left(-\Delta, f_{1}, q_{2}, \mathscr{B}\right)$. Since $f \geqq f_{1}$, we have

$$
\int_{\mathscr{B}} F\left(x, \psi_{4}-q_{2}\right) \geqq \int_{\mathscr{B}} F_{1}\left(\psi_{4}-q_{2}\right)=\mu ;
$$

hence $t \psi_{4} \in S\left(f, q_{2}, \mathscr{B}\right)$ for some $t \in(0,1]$, and so $\left\|\psi_{3}\right\|^{2} \leqq\left\|t \psi_{4}\right\|^{2} \leqq\left\|\psi_{4}\right\|^{2}$.

Combining these inequalities, we have $B(\psi) \leqq \beta_{2}\left\|\psi_{4}\right\|^{2}$, and $\left\|\psi_{4}\right\|^{2}$ is bounded by the estimate (C.10), in Appendix C, for spherically symmetrical cases; this estimate differs only slightly from (3.5).

(ii) For any closed set $K \subset \Omega$, we define the electrostatic capacity of $K$ relative to $\Omega$ by

$$
\operatorname{cap}(K, \Omega)=\min _{u \in W,\left.u\right|_{K} \geqq 1}\|u\|^{2},
$$


where $\left.u\right|_{K} \geqq 1$ means that there exists a sequence $\left\{u_{n}\right\}$ in $C_{0}^{\infty}(\Omega)$ such that $u_{n}(x) \geqq 1$ on $K$, and $u_{n} \rightarrow u$ in $W$. The infimum is attained, in view of the lemma of projection onto a closed, convex (non-empty) set in a Hilbert space; for further details, see [14], p. 147. In the present case, we choose $K=\bar{A}$ and note that $\psi / q_{1}$ is admitted to the capacity competition for $\bar{A}$; consequently,

$$
\operatorname{cap}(\bar{A}, \Omega) \leqq\|\psi\|^{2} / q_{1}(\mu)^{2} \leqq B(\psi) / \beta_{1} q_{1}(\mu)^{2} .
$$

On the other hand, when $K$ is connected there is a lower bound [7] for $\operatorname{cap}(K, \Omega)$ in terms of the ratio $\operatorname{diam} K / \operatorname{diam} \Omega$. In the present case, $\bar{A}$ is connected because $A$ is, and $\operatorname{diam} \bar{A}=\operatorname{diam} A$, so that

$$
\operatorname{cap}(\bar{A}, \Omega)>2 \pi / \sinh ^{-1} \frac{1}{\delta} \quad(\delta=\operatorname{diam} A / \operatorname{diam} \Omega) .
$$

[A lower bound sharper than (4.14) is also given in [7], but (4.14) is simpler than the sharp estimate, and is asymptotically equal to it as $\delta \rightarrow 0$.]

Combining (4.13) and (4.14), we obtain

$$
\delta<1 / \sinh \left\{2 \pi \beta_{1} q_{1}(\mu)^{2} / B(\psi)\right\},
$$

and, in view of (2.10) and (4.9), the estimate (4.10) follows.

(iii) Recall that $\Psi=\psi-q$ and that

$$
\lambda(\mu)=B(\psi) / \int_{\Omega} \psi f(x, \Psi) ;
$$

hence the upper bound (4.9) for $B(\psi)$, and a lower bound for the integral in the denominator of (4.15), yield an upper bound for $\lambda$. Now $f(x, t)$ is a non-decreasing function of $t$, so that $F(x, t) \leqq t f(x, t)$ for $t \geqq 0$; consequently, (4.11) follows from the estimate

$$
\int_{\Omega} \psi f(x, \Psi)=\int_{\Omega}(\Psi+q) f(x, \Psi)>\int_{\Omega} F(x, \Psi)=\mu .
$$

(iv) We now seek an upper bound for the denominator in (4.15). By (2.9) and (2.10),

$$
\begin{aligned}
\int_{\Omega} \psi f(x, \Psi) & \leqq \int_{A}\left(\Psi+q_{2}\right)\left(K_{2} \Psi+M_{2} \Psi^{m_{2}}\right) \\
& =K_{2} \int_{A}\left(\Psi^{2}+q_{2} \Psi\right)\left(1+\frac{M_{2}}{K_{2}} \Psi^{2 l}\right),
\end{aligned}
$$

where $l=\frac{1}{2}\left(m_{2}-1\right)$. Let $d=\operatorname{diam} A$; then $A$ is contained in a square with sides of length $d$. Modifying a calculation by Nirenberg ([11], p. 128; the context is Sobolev embedding), we find that, since $\left.\Psi\right|_{\partial A}=0$,

$$
\int_{A} \Psi^{p} \leqq c_{p} d^{2}\left\{\int_{A}|\nabla \Psi|^{2}\right\}^{p / 2}, \quad 1 \leqq p<\infty
$$

where $c_{p}=2^{-3 / 2}$ if $1 \leqq p \leqq 2$, and $c_{p}=2^{-5 / 2} p$ if $p \geqq 2$.

Now, with $\Psi_{+}(x)=\max \{\Psi(x), 0\}$, we have $\Psi_{+} \in W([10]$, p. 50) and hence

$$
\int_{A}|\nabla \Psi|^{2}=\int_{\Omega}\left|\nabla \Psi_{+}\right|^{2} \leqq \frac{1}{\beta_{1}} B\left(\Psi_{+}\right)
$$


by (2.8). But, by (4.3), $B\left(\Psi_{+}\right)=B_{A}(\Psi) \leqq B_{A}(\psi)<B(\psi)$, so that

$$
\int_{A} \Psi^{p}<k_{p} d^{2} B(\psi)^{p / 2}, \quad k_{p}=c_{p} \beta_{1}^{-p / 2}, \quad 1 \leqq p<\infty,
$$

and, by (4.16),

$$
\begin{aligned}
& \int_{\Omega} \psi f(x, \psi)<K_{2} d^{2} \\
& \quad \cdot\left\{k_{2} B(\psi)+k_{1} q_{2} B(\psi)^{1 / 2}+\frac{M_{2}}{K_{2}}\left[k_{2 l+2} B(\psi)^{l+1}+k_{2 l+1} q_{2} B(\psi)^{l+1 / 2}\right]\right\} .
\end{aligned}
$$

Since $k_{1} / k_{2}=\beta_{1}^{1 / 2}$, we obtain from (4.15)

$$
\frac{1}{\lambda}<\operatorname{const} \delta^{2}\left\{1+\beta_{1}^{1 / 2} q_{2} B(\psi)^{-1 / 2}\right\}\left\{1+\text { const } B(\psi)^{l}\right\}
$$

finally, (4.13) and (4.14) imply that

$$
\beta_{1}^{1 / 2} q_{2} B(\psi)^{-1 / 2}<\gamma_{2}\left(\frac{1}{2 \pi} \sinh ^{-1} \frac{1}{\delta}\right)^{1 / 2},
$$

and this proves (4.12).

\section{Nonlinear Desingularization: The Limiting Behaviour as $\boldsymbol{\mu} \rightarrow \mathbf{0}$}

We are now in a position to make good the heuristic ideas of Sect. 3.

Lemma 5.1. Let the Green function $G$ be as in (3.1); then, for any points $x$ and $x_{0}$ in $\Omega$,

$$
\int_{\Omega}\left|\nabla_{z}\left\{G(z, x)-G\left(z, x_{0}\right)\right\}\right|^{p} d z \leqq \text { const }\left|x-x_{0}\right|^{2-p}\left(1+\log \frac{D}{\left|x-x_{0}\right|}\right)^{2},
$$

where $1 \leqq p<2, D=\operatorname{diam} \Omega$, and the constant depends only on $L, \Omega$ and $p$.

Proof. (i) Suppose for the moment that $\Omega$ is a bounded domain in $\mathbb{R}^{N}$ with $N \geqq 2$, the operator $L$ and boundary $\partial \Omega$ being restricted as before in all other respects. Let $|z-x|=r$; the estimates

$$
\left|\nabla_{z} G(z, x)\right| \leqq \text { const } r^{-N+1}, \quad\left|\nabla_{z} \nabla_{x} G(z, x)\right| \leqq \text { const } r^{-N}
$$

are proved in [16] for $N \geqq 3$, under hypotheses on $L$ and $\partial \Omega$ that are weaker than ours. These estimates are true also for $N=2$ when $-L$ is the Laplace operator [several references are given by Kato, T.: Arch. Ration. Mech. Anal. 25, 190 (1967)]. Professor Widman has kindly written to us that (A) holds also in the present case $\left(N=2\right.$, with smooth coefficients $a_{i j}$ in $L$, and smooth boundary $\left.\partial \Omega\right)$, but that the proof involves a "rather ugly argument". If the proofs in [16] are repeated for $N=2$, there results

$$
\begin{array}{r}
\left|\nabla_{z} G(z, x)\right| \leqq \text { const } r^{-1}\left(1+\log \frac{D}{r}\right), \\
\left|\nabla_{z} \nabla_{x} G(z, x)\right| \leqq \text { const } r^{-2}\left(1+\log \frac{D}{r}\right),
\end{array}
$$

and it is these estimates that we shall use here. 
(ii) Let $|z-x|=r,\left|z-x_{0}\right|=r_{0}$ and $\left|x-x_{0}\right|=\delta$; abbreviate $G(z, x)$ to $G$, and $G\left(z, x_{0}\right)$ to $G_{0}$. We may and shall assume that $3 \delta \leqq D$.

For $r<2 \delta$, we use (B) and add the contributions of $G$ and $G_{0}$. If $p=1$, we have

$$
\begin{aligned}
\int_{\Omega \cap\{r<2 \delta\}}\left|\nabla_{z}\left\{G-G_{0}\right\}\right| d z & \leqq \text { const }\left\{\int_{0}^{2 \delta}\left(1+\log \frac{D}{r}\right) d r+\int_{0}^{3 \delta}\left(1+\log \frac{D}{r_{0}}\right) d r_{0}\right\} \\
& \leqq \text { const } \delta\left(1+\log \frac{D}{\delta}\right),
\end{aligned}
$$

while, if $1<p<2$,

$$
\begin{aligned}
& \int_{\Omega \cap\{r<2 \delta\}}\left|\nabla_{z}\left\{G-G_{0}\right\}\right|^{p} d z \\
& \leqq \text { const }\left\{\int_{0}^{2 \delta} r^{1-p}\left(1+\log \frac{D}{r}\right)^{2} d r+\int_{0}^{3 \delta} r_{0}^{1-p}\left(1+\log \frac{D}{r_{0}}\right)^{2} d r_{0}\right\} \\
& \leqq \text { const } \delta^{2-p}\left(1+\log \frac{D}{\delta}\right)^{2} .
\end{aligned}
$$

(iii) For $r \geqq 2 \delta$, integration of second derivatives from $x_{0}$ to $x$ gives

$$
\left|\nabla_{z}\left\{G-G_{0}\right\}\right| \leqq \operatorname{const} \delta r^{-2}\left(1+\log \frac{D}{r}\right) .
$$

Then, if $p=1$,

$$
\begin{aligned}
\int_{\Omega \cap\{r \geqq 2 \delta\}}\left|\nabla_{z}\left\{G-G_{0}\right\}\right| d z & \leqq \text { const } \delta \int_{2 \delta}^{D} r^{-1}\left(1+\log \frac{D}{r}\right) d r \\
& =\operatorname{const} \delta \log \frac{D}{2 \delta}\left(1+\frac{1}{2} \log \frac{D}{2 \delta}\right),
\end{aligned}
$$

while, if $1<p<2$,

$$
\begin{aligned}
\int_{\Omega \cap\{r \geqq 2 \delta\}}\left|\nabla_{z}\left\{G-G_{0}\right\}\right|^{p} d z & \leqq \operatorname{const} \delta^{p} \int_{2 \delta}^{D} r^{1-2 p}\left(1+\log \frac{D}{r}\right)^{2} d r \\
& \leqq \operatorname{const} \delta^{2-p}\left(1+\log \frac{D}{\delta}\right)^{2} .
\end{aligned}
$$

The lemma is proved.

Theorem 5.2. With $\psi$ denoting the solution of the variational problem (2.6), and with $\Psi=\psi-q$, define

$$
h(\mu)=\lambda \int_{A} f(x, \Psi),
$$

and let $a(\mu)$ be any point of the set $A(\mu)=\{x \mid \Psi(x, \mu)>0\}$. Then, as $\mu \rightarrow 0$,

$$
\frac{\psi(\cdot, \mu)}{h(\mu)}-G(\cdot, a(\mu)) \rightarrow 0 \quad \text { in } \quad \stackrel{\circ}{W}_{p}^{1}(\Omega), \quad 1 \leqq p<2,
$$

and hence in $L_{r}(\Omega), 1 \leqq r<\infty$. 
Proof. The representation (in which dependence on $\mu$ is now implied)

$$
\psi(z)=\lambda \int_{A} G(z, x) f(x, \Psi) d x
$$

follows from (2.5) in the usual way (for example, as in [8], p. 47) if we choose the test function $\phi$ in (2.5) to be a smooth approximation to $G$ and then pass to the limit. Since

by (5.1), we have

$$
\frac{\lambda}{h(\mu)} \int_{A} f(x, \Psi)=1
$$

$$
\frac{\psi(z)}{h(\mu)}-G(z, a)=\frac{\lambda}{h(\mu)} \int_{A}\{G(z, x)-G(z, a)\} f(x, \Psi) d x .
$$

We form the $L_{p}$ norm of the gradient of this function, and apply the Minkowski inequality, to obtain

$$
\begin{aligned}
\left\|\frac{\psi}{h(\mu)}-G(\cdot, a)\right\|_{1, p} \leqq & \frac{\lambda}{h(\mu)} \int_{A} f(x, \Psi) d x \\
& {\left[\int_{\Omega}\left|\nabla_{z}\{G(z, x)-G(z, a)\}\right|^{p} d z\right]^{1 / p} . }
\end{aligned}
$$

Here $x$ and $a$ are both in $A$; by Theorem 4.4 and the remark following it,

$$
|x-a| \leqq \operatorname{diam} A<\operatorname{const} \varrho(\mu)^{c}, \quad c>0,
$$

where $\varrho(\mu) \rightarrow 0$ as $\mu \rightarrow 0$, as is shown by (4.5). Lemma 5.1 now shows that in (5.4) the function in square brackets tends to zero uniformly over $x \in A$, and the remaining expression is bounded, by (5.3). Convergence to zero in $L_{r}(1 \leqq r<\infty)$ follows from Sobolev embedding.

Remarks. 1 . Since regularity theory shows that $\psi(x, \mu) / h(\mu)$ is bounded pointwise at fixed $\mu$, while $G(\cdot, a(\mu)) \in \stackrel{\circ}{W}_{p}^{1}(\Omega)$ only for $p<2$, the result is the best possible of its kind.

2. An upper bound for $h(\mu)$ is easily obtained. By (2.7), and since $\psi(x)>q_{1}(\mu)$ in A,

$$
h(\mu)=\lambda \int_{A} f(x, \Psi)=B(\psi) \int_{A} f(x, \Psi) / \int_{A} \psi f(x, \Psi)<B(\psi) / q_{1}(\mu)
$$

hence the bound (4.9) on $B(\psi)$ implies that

$$
h(\mu) \leqq c_{2} q_{2}(\mu) / g(\mu), \quad c_{2}=2 \pi \beta_{2} \gamma_{2} .
$$

It follows from (4.7) that, if

$$
q(x, \mu)=o\left(\log \frac{1}{\mu}\right) \text { as } \mu \rightarrow 0,
$$

as is certainly the case when $q_{0}$ is fixed (is independent of $\mu$ ), then $h(\mu) \rightarrow 0$ and so $\psi(\cdot, \mu) \rightarrow 0$ in $W_{p}^{1}(\Omega), 1 \leqq p<2$. In that case (5.2) still shows $\psi$ to be asymptotically proportional to the Green function, but the result seems more interesting if $q$ is allowed to vary in such a way that $h(\mu) \nrightarrow 0$.

The condition (5.6) is sharp in the sense that, for the spherically symmetric cases in Appendix $\mathrm{C}$, the function $h(\mu)$ tends to a positive limit when $q(\mu)$ $\sim$ const $\log (1 / \mu)$ as $\mu \nrightarrow 0$. 
Theorem 5.3. As in (4.4), (4.10), and (4.12), define $k=\frac{1}{2}\left(m_{1}+1\right), l=\frac{1}{2}\left(m_{2}-1\right)$ and $c=\beta_{1} / \beta_{2} \gamma_{2}^{2}$. Assume that $q_{2}(\mu) \leqq$ const $\mu^{-r}$, where $r$ is any positive number if $k c \geqq l$, or $0<r<\frac{1}{2} c(l-k c)^{-1}$ if $k c<l$. Then $\lambda(\mu) \rightarrow \infty$ as $\mu \rightarrow 0$.

Proof. In view of the upper bound (4.12) for $1 / \lambda$, the only question is whether

$$
\delta^{2}\left(\sinh ^{-1} \frac{1}{\delta}\right)^{1 / 2} B(\psi)^{l} \rightarrow 0 .
$$

Let $\Lambda(\mu)$ be a generic symbol for functions that are $O\left(|\log \mu|^{b}\right)$ for some real number $b$, as $\mu \rightarrow 0$. By (4.10) and (4.4)-(4.6),

while

$$
\delta \leqq \text { const } \varrho(\mu)^{c} \leqq \mu^{c / 2} q_{2}(\mu)^{-k c} \Lambda(\mu)
$$

by (4.9). Accordingly,

$$
B(\psi) \leqq q_{2}(\mu)^{2} \Lambda(\mu)
$$

$$
\delta^{2}\left(\sinh ^{-1} \frac{1}{\delta}\right)^{1 / 2} B(\psi)^{l} \leqq \mu^{c} q_{2}(\mu)^{2(l-k c)} \Lambda(\mu),
$$

which implies the result. [For the case $k c \geqq l$, we recall that $q_{2}(\mu) \geqq \gamma_{1}>0$.]

\section{Appendix A.}

\section{Comparison with the Theory of Steady Vortex Rings}

The problem of steady vortex rings, in a form analogous to (2.2), may be stated as follows. Let $(r, \theta, z)$ be cylindrical co-ordinates in $\mathbb{R}^{3}$, let $\Pi=\{(r, z) \mid r>0\}$ denote a meridional half-plane ( $\theta=$ const), and let $\Omega \subset \Pi$ be a domain. We seek a Stokes stream function $\Psi$ (which means that the fluid velocity has components $-r^{-1} \partial \Psi / \partial z, 0, r^{-1} \partial \Psi / \partial r$ in the directions $r, \theta, z$ increasing) such that

$$
\begin{aligned}
L \Psi & \equiv-\left\{\frac{\partial}{\partial r}\left(\frac{1}{r} \frac{\partial}{\partial r}\right)+\frac{1}{r} \frac{\partial^{2}}{\partial z^{2}}\right\} \Psi=\lambda r f_{0}(\Psi) \text { in } \Omega, \\
\left.\Psi\right|_{\partial \Omega} & =-\frac{1}{2} W r^{2}-k,
\end{aligned}
$$

where $f_{0}(t)=0$ for $t \leqq 0$, and $f_{0}(t)>0$ for $t>0$, while $W>0$ and $k$ are prescribed constants. We write $x=(r, z)$.

In [8], $\Omega$ is initially the rectangle $D=(0, a) \times(-b, b)$, and finally $\Omega=\Pi$ is admitted; $f_{0}$ is initially Hölder continuous, and is finally allowed a simple discontinuity at the origin; and $k$ is merely non-negative. Thus the problem in [8] violates every hypothesis in the present paper: the coefficients $a_{i j}$ are not in $C^{\infty}(\bar{D})$, since $a_{11}(x)=a_{22}(x)=1 / r$; the boundary $\partial D$ has corners and hence is not of class $C^{\infty}$; the domain $\Omega$ may be unbounded; $f(x, t)=r f_{0}(t)$ is much less restricted than here; and zero boundary values of $\Psi$ are allowed on a part of $\partial \Omega$.

However, if in (A.1) we take $\Omega$ to be a bounded domain in which $r>$ const $>0$, and $\partial \Omega$ to be of class $C^{\infty}$, if we restrict $f(x, t)=r f_{0}(t)$ as in Sect. 2.4, and if we so choose $k$ that $-\frac{1}{2} W r^{2}-k<0$ on $\partial \Omega$, then all the present hypotheses are satisfied, and there remains a problem which is physically significant and quite general enough for our main purpose here: to study the degeneration of a steady vortex ring to a singular vortex circle. 
In the perturbation theory of steady vortex rings of small cross-section [6] one prescribes $\lambda, f_{0}$ and the approximate area and location of the cross-section $A$ in such a way that (a) the velocity $W$ and flux constant $k$ have to be found a posteriori, (b) the circulation $h=\lambda \int_{A} r f_{0}(\Psi)$ tends to a positive limit as $d=\operatorname{diam} A \rightarrow 0$. It turns out that $W$ and $k$ both tend to infinity like $\log (1 / d)$, which is consistent with the present results.

It has not yet been proved (as far as we are aware) that the steady vortex rings constructed by the perturbation theory are solutions of the variational problem formulated for the global theory. The present paper represents a useful step in this direction because, starting with a variational principle equivalent to that in [8], we have found properties of the solution that agree with those of solutions established by the perturbation theory. In fact, if the sets $A$ of the present approach could be proved to be asymptotically circular, for the problem (A.1), then the local uniqueness of the perturbation solutions could be used to identify the two types of solution.

For the problem of steady planar vortex pairs [12], similar remarks apply, except that the operator $L$ is now the two-dimensional Laplacian, so that singular coefficients do not occur.

\section{Appendix B. Equivalence of two Variational Principles ${ }^{1}$}

Let $\mathbb{R}_{+}=[0, \infty)$. In this appendix we consider the functions $M: \mathbb{R}_{+} \times I \rightarrow \mathbb{R}_{+}$and $m: \mathbb{R}_{+} \times I \rightarrow \mathbb{R}_{+}$defined by

$$
M(\eta, \kappa)=\max _{B(u)=\eta} J(u, \kappa) \text { and } m(\mu, \kappa)=\min _{J(u, \kappa)=\mu} B(u),
$$

it being understood that $J(\cdot, \kappa)$ and $B$ are defined on $W$. Our conclusions are for each fixed value of $\kappa$; therefore we abbreviate $M(\eta, \kappa)$ to $M(\eta)$, and similarly for $J$ and $m$.

It is clear that $M(0)=0$, because $B(u)=0$ implies that $u=0$ in $W$, by (2.8), and that $m(0)=0$. The existence of $M(\eta)$ for $\eta>0$ follows from a proof precisely like that of Theorem 3A in [8], and the existence of $m(\mu)$ for $\mu>0$ follows from Theorem 4.1 of the present paper; both functions are strictly positive on $(0, \infty)$.

In the present context, the function $f$ need not be $C^{1}$ and convex in its second argument. It is sufficient that $f$ be Hölder continuous; that $f(x, t)=0$ for $t \leqq 0$ and $f(x, t)>0$ for $t>0$; that $f(x, t)$ be non-decreasing in $t$; and that $f(x, t)=O\left(t^{m_{2}}\right)$ as $t \rightarrow \infty$ (for some $m_{2} \geqq 0$ ) uniformly over $x \in \bar{\Omega}$.

We shall use repeatedly, without further comment, the fact that $F(x, t)$ is a strictly increasing function of $t$ for $t \geqq 0$, so that $u_{2}(x)>u_{1}(x)$ and $J\left(u_{1}\right)>0$ imply that $J\left(u_{2}\right)>J\left(u_{1}\right)$.

Theorem B.1. The functions $M$ and $m$ are inverses of each other:

$$
\begin{aligned}
& \eta=m(M(\eta))=\min _{J(u)=M(\eta)} B(u), \quad \forall \eta \in \mathbb{R}_{+}, \\
& \mu=M(m(\mu))=\max _{B(u)=m(\mu)} J(u), \quad \forall \mu \in \mathbb{R}_{+} .
\end{aligned}
$$

Accordingly, $M$ and $m$ are surjective (onto $\mathbb{R}_{+}$).

1 This appendix represents joint work with Grant Keady 
Remark. Note the following implication of (B.1): a maximizer, say $\phi$, of $J(u)$ over the set $B(u)=\eta$ also minimizes $B(u)$ over the set $J(u)=M(\eta)$, since $J(\phi)=M(\eta)$ and $B(\phi)=\eta$. A similar remark applies to (B.2). Therefore, the solution sets

and

$$
\{\phi \in W \mid \exists \eta \geqq 0 \text { such that } B(\phi)=\eta \text { and } J(\phi)=M(\eta)\}
$$

$$
\{\psi \in W \mid \exists \mu \geqq 0 \text { such that } J(\psi)=\mu \text { and } B(\psi)=m(\mu)\}
$$

of the two variational problems (all $\eta \geqq 0$ and $\mu \geqq 0$ being considered) are one and the same, each being included in the other.

Proof of Theorem B.1. Because there exists a maximizer $\phi$ such that $J(\phi)=M(\eta)$ and $B(\phi)=\eta$, it is sufficient for (B.1) to prove that the minimum there is not less than $\eta$. Suppose that $\eta>0$ [otherwise (B.1) is obvious] and that $J(v)=M(\eta)$ and $B(v)<\eta$ for some $v \in W$. Define $\hat{v}=\{\eta / B(v)\}^{1 / 2} v$; then $B(\hat{v})=\eta$ and $J(\hat{v})>J(v)=M(\eta)$, which contradicts the definition of $M$.

Similarly, it is sufficient for (B.2) to prove that the maximum there is not greater than $\mu$. Suppose that $\mu>0$ [otherwise (B.2) is obvious] and that $B(w)=m(\mu)$ and $J(w)>\mu$ for some $w \in W$. Then for some $t \in(0,1)$ we have $J(t w)=\mu$ and $B(t w)$ $<B(w)=m(\mu)$, which contradicts the definition of $m$.

Finally, (B.1) shows that $m$ is surjective, and (B.2) that $M$ is.

Theorem B.2. The functions $M$ and $m$ are strictly increasing and continuous.

Proof. To prove that $M$ is strictly increasing, let $0 \leqq \eta_{1}<\eta_{2}$. If $\eta_{1}=0$, one constructs a function $v$ such that $B(v)=\eta_{2}$ and $J(v)>0$; hence $M\left(\eta_{2}\right)>M\left(\eta_{1}\right)=0$. If $\eta_{1}>0$, let $\phi_{1}$ be a maximizer of $J(u)$ over the set $B(u)=\eta_{1}$, and define $v_{2}=\left(\eta_{2} / \eta_{1}\right)^{1 / 2} \phi_{1}$. Then $B\left(v_{2}\right)=\eta_{2}$ and $J\left(v_{2}\right)>J\left(\phi_{1}\right)=M\left(\eta_{1}\right)$; hence $M\left(\eta_{2}\right)>M\left(\eta_{1}\right)$.

For the function $m$, and $0 \leqq \mu_{1}<\mu_{2}$, we use a minimizer, say $\psi_{2}$, of $B(u)$ over the set $J(u)=\mu_{2}$, and consider $t \psi_{2}$ where $t \in(0,1)$ and is such that $J\left(t \psi_{2}\right)=\mu_{1}$.

The functions $M$ and $m$ are continuous because they are increasing and surjective.

\section{Appendix C. Some Spherically Symmetric Cases}

\section{C.1. Reduction of the Problem}

Here we consider the following particular form of the problem (2.2): $\Omega$ is the ball (or disk) $\mathscr{B}$ of radius $b$ about the origin, $L=-\Delta$ (where $\Delta$ denotes the Laplace operator), $q(x, \kappa)=q(\kappa)$ and $f(x, t)=M t^{m}$ for $t \geqq 0$, with $M>0$ and $m>1$. Then the problem (2.2) certainly has spherically symmetric solutions, and symmetrization with respect to a point $([13]$, p. 63$)$ proves the same for the variational principle (2.6).

In place of $\Psi(x)$, we use the notation $\tilde{V}(x)=V(r), r=|x|$, for the present solution. By Theorem 4.1, we have $\tilde{V} \in C^{2+\alpha}(\overline{\mathscr{B}}), J(\tilde{V}+q)=\mu>0$ and

$$
\left.\begin{array}{rl}
V_{r r}+\frac{1}{r} V_{r} & =-\lambda(\mu, \kappa) M V_{+}^{m} \quad \text { in } \quad(0, b), \\
\left.V\right|_{r=b} & =-q(\kappa)<0,
\end{array}\right\}
$$


where $V_{r}=\partial V / \partial r$ and $V_{+}(r)=\max \{V(r), 0\}$. By the maximum principle, the set $A=\{x \mid \tilde{V}(x)>0\}$ must be a ball about the origin (otherwise $V$ would have an interior local minimum); let its radius be $a$. Then for $r \geqq a$

$$
V(r)=-q(\kappa) \log \frac{r}{a} / \log \frac{b}{a}, \quad a \leqq r \leqq b .
$$

If we make the transformation

$$
\Phi(s)=V(r) / V(0), \quad s=V(0)^{\frac{m-1}{2}}(\lambda M)^{1 / 2} r,
$$

then Theorem 4.1 and (C.1) imply that

$$
\left.\begin{array}{c}
\Phi_{s s}+\frac{1}{S} \Phi_{s}=-\Phi_{+}^{m}, \quad 0<s<s(b), \\
\Phi(0)=1, \quad \Phi_{s}(0)=0 .
\end{array}\right\}
$$

Conversely, we can infer results about $V$ from (C.3) and (C.4). Let (C. $4^{\prime}$ ) denote the initial-value problem resulting from (C.4) when we remove the condition $s<s(b)$, and specify that the differential equation need not hold at a zero of $\Phi$ (in fact, $\Phi$ has only one zero), but that $\Phi_{s}$ must be continuous there. It is a matter of some length, but little difficulty, to prove

Lemma C.1. The initial-value problem (C.4') has a unique solution $\Phi$ on any interval $[0, N]$ not merely for $m>1$, but for $m \geqq 0$; and $\Phi \in C^{2}[0, N]$ if $m>0$, or is piecewise $C^{2}$ (with a simple discontinuity of $\Phi_{s s}$ at the zero of $\Phi$ ) if $m=0$.

\section{C.2. Dependence on the Parameters}

In view of Lemma C.1, we may regard $\Phi$ as known; $K_{j}$ will denote positive numbers depending only on $\Phi$ (hence only on $m$ ), and we also write

$$
\varrho=\varrho(\mu, \kappa)=a / b, \quad q=q(\kappa) .
$$

Calculations based on (C.2), (C.3) and the continuity of $V_{r}$ at $r=a$, yield the following.

$$
\begin{aligned}
V(0) & =K_{0} q / \log \frac{1}{\varrho}, \\
\frac{1}{\lambda M} & =K_{1} b^{2} \varrho^{2}\left(q / \log \frac{1}{\varrho}\right)^{m-1}, \\
\|\tilde{V}\|^{2} & =\|\tilde{V}+q\|^{2}=\frac{2 \pi q^{2}}{\log (1 / \varrho)}\left\{1+\frac{K_{2}}{\log (1 / \varrho)}\right\}, \\
\mu & =\frac{M}{m+1} \int_{\mathscr{B}} \tilde{V}_{+}^{m+1}=K_{3} M b^{2} \varrho^{2}\left(q / \log \frac{1}{\varrho}\right)^{m+1}, \\
h(\mu, \kappa) & =\lambda \int_{\mathscr{B}} \tilde{V}_{+}^{m}=2 \pi q / \log \frac{1}{\varrho} .
\end{aligned}
$$

To solve (C.8) for $\varrho(\mu, \kappa)$, we use the elementary but useful 
Lemma C.2. The equation

$$
t\left(\log \frac{1}{t}\right)^{-k}=\varepsilon, \quad 0<t<1, \quad k>0, \quad \varepsilon>0,
$$

has a unique solution $t_{*}(\varepsilon)$. If $\varepsilon<1 / e$ and $\varepsilon\left(\log \varepsilon^{-1}\right)^{k}<1$ then

$$
\varepsilon\left(\log \frac{1}{\varepsilon}-k \log \log \frac{1}{\varepsilon}\right)^{k}<t_{*}(\varepsilon)<\varepsilon\left(\log \frac{1}{\varepsilon}\right)^{k} .
$$

Proof. Let $t=1 / x$ and $\varepsilon=1 / \eta$; the given equation becomes

$$
\phi(x) \equiv x(\log x)^{k}=\eta, \quad x>1, \quad \eta>0 .
$$

Since $\phi$ is strictly increasing and has range $(0, \infty)$, this has a unique solution $x_{*}(\eta)$. Let

$$
x_{1}=\eta(\log \eta)^{-k} \quad \text { and } \quad x_{2}=\eta(\log \eta-k \log \log \eta)^{-k}
$$

then, if $\eta>e$ (so that $\log \log \eta>0$ ) and $x_{1}>1$, we have $\phi\left(x_{1}\right)<\eta$, which implies that $x_{1}<x_{*}(\eta)$, and $\phi\left(x_{2}\right)>\eta$, which implies that $x_{2}>x_{*}(\eta)$.

Remarks. 1. We note that, by (C.2),

$$
V(r)+q(\kappa)=q(\kappa) \log \frac{b}{r} / \log \frac{b}{a}=h(\mu, \kappa) G(x, 0), \quad a \leqq r \leqq b,
$$

where $h$ is as in (C.9), and $G(x, y)$ is the Green function of $-\Delta$ in $\mathscr{B}$; also, the radius $a=b \varrho(\mu, \kappa) \rightarrow 0$ as $\mu \rightarrow 0$. It is this kind of result that we generalize in Theorem 5.2.

2. Setting $\kappa=\mu$, we ask whether we can so choose $q(\mu)$ that the function $h$ in (C.9) is a constant. It follows easily from (C.8) and (C.9) that $h(\mu)=$ const if, and only if,

$$
q(\mu)=\frac{c}{K} \log \left(c^{k} \mu^{-1 / 2}\right), \quad k=\frac{1}{2}(m+1), \quad K=\left(K_{3} M b^{2}\right)^{1 / 2 k},
$$

where $c$ is an arbitrary positive constant; then $h(\mu)=2 \pi c / K$. [Correspondingly, if $q(\mu) \sim$ const $\log (1 / \mu)$ as $\mu \rightarrow 0$, then $h(\mu)$ tends to a positive limit.] In the perturbation theory of steady vortex rings [6], one prescribes (in effect) a fixed value of the circulation, which corresponds to the present $h(\mu)$, and again finds that the analogue of $q(\mu)$ is asymptotically proportional to $\log (1 / \mu)$ as $\mu \rightarrow 0$.

\section{C.3. An Explicit Upper Bound for $\|\tilde{V}\|$}

Since the constants $K_{2}(m)$ and $K_{3}(m)$ in (C.7) and (C.8) are not known explicitly, we bound $\|\tilde{V}\|$ by means of the trial function

$$
U(r)=\left\{\begin{array}{l}
p\left(1-\frac{r^{2}}{a^{2}}\right), \quad r \leqq a, \\
-q \log \frac{r}{a} / \log \frac{b}{a}, \quad a \leqq r \leqq b,
\end{array}\right.
$$


where $p$ and $a$ are now positive parameters which we choose as follows. First the condition $J(\tilde{U}+q)=\mu$ determines $p=p(a)$, then we minimize $\|\tilde{U}\|$ with respect to $a$. There results

$$
\|\tilde{V}\|^{2} \leqq\|\tilde{U}\|^{2}=\frac{2 \pi q^{2}}{\log (1 / \varrho)}\left\{1+\frac{k}{2 \log (1 / \varrho)}\right\}, \quad k=\frac{1}{2}(m+1), \quad \varrho=\frac{a}{b},
$$

where $\varrho=\varrho(\mu, \kappa)$ is now the solution of

$$
\varrho\left(\log \frac{1}{\varrho}\right)^{-k}=C \mu^{1 / 2} q(\kappa)^{-k}, \quad C=\frac{1}{b}\left(\frac{2}{k}\right)^{k / 2}\left\{\frac{2 k(2 k+1)}{M \pi}\right\}^{1 / 2} .
$$

Note that (C.10) and (C.11) have precisely the same form as (C.7) and (C.8) (possibly because the trial function $U$ has the form of the exact solution for $m=0$ ); only the constants differ.

\section{References}

1. Abrikosov, A.A.: On the magnetic properties of superconductors of the second group. Sov. Phys. JETP 5, 1174-1182 (1957)

2. Adler, S.L.: Global structure of static Euclidean SU(2) solutions. Phys. Rev. D 20, 1386-1411 (1979)

3. Agmon, S., Douglis, A., Nirenberg, L.: Estimates near the boundary for solutions of elliptic partial differential equations satisfying general boundary conditions. I. Comm. Pure Appl. Math. 12, 623-727 (1959)

4. Berger, M.S.: A bifurcation theory for nonlinear elliptic partial differential equations. In: Bifurcation theory and nonlinear eigenvalue problems (eds. J. B. Keller, S. Antman), pp. 113-216. New York: Benjamin 1969

5. Berger, M.S.: Nonlinearity and functional analysis. New York: Academic Press 1977

6. Fraenkel, L.E. : On steady vortex rings of small cross-section in an ideal fluid. Proc. R. Soc. London A 316, 29-62 (1970)

7. Fraenkel, L.E.: A lower bound for electrostatic capacity in the plane. Proc. R. Soc. Edinburgh, Sect. A, to appear

8. Fraenkel, L.E., Berger, M.S.: A global theory of steady vortex rings in an ideal fluid. Acta Math. 132, 13-51 (1974)

9. Keady, G., Norbury, J.: A semilinear elliptic eigenvalue problem. I, II. Proc. R. Soc. Edinburgh, A, to appear

10. Ladyzhenskaya, O.A., Ural'tseva, N.N.: Linear and quasilinear elliptic equations. New York: Academic Press 1968

11. Nirenberg, L.: On elliptic partial differential equations. Ann. Scuola Norm. Sup. Pisa (3) 13, 115-162 (1959)

12. Norbury, J.: Steady planar vortex pairs in an ideal fluid. Commun. Pure Appl. Math. 28, 679-700 (1975)

13. Pólya, G., Szegö, G.: Isoperimetric inequalities in mathematical physics. Princeton: Princeton University Press 1951

14. Stampacchia, G.: Variational inequalities. Theory and applications of monotone operators. Proc. NATO Advanced Study Inst., pp. 101-192. Gubbio: Ediz. Oderisi 1969

15. Temam, R.: A non-linear eigenvalue problem: the shape at equilibrium of a confined plasma. Arch. Ration. Mech. Anal. 60, 51-73 (1976)

16. Widman, K.-O. : Inequalities for Green functions of second order elliptic operators. Report 8-1972, Dept. of Math., Linköping University, Sweden 1972

Communicated by J. Glimm

Received October 16, 1979 